\title{
BUNDLES ALONG THE FIBER IN THE PL CATEGORY
}

\author{
NORMAN LEVITT
}

\author{
(Communicated by Haynes R. Miller)
}

\begin{abstract}
We show that the notion of the bundle of tangents along the fiber over the total space of a fibering is not a good one when the fibering in question is PL. In particular it is shown that there are vector bundles over the same base space such that (1) the associated sphere bundles are PL-equivalent; (2) the consequent homeomorphism of total spaces is not covered by any PL-disk bundle equivalence of the respective bundles of tangents to the fiber manifolds.
\end{abstract}

A familiar construction in differential topology is that of the bundle of tangents along the fiber for a fibering whose fibers are smooth manifolds and whose structure group is a group of diffeomorphisms of the fiber. Thus if

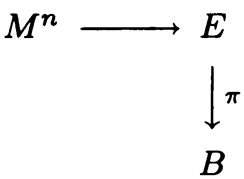

is such a fibering, then there is an $n$-vector bundle $V$ over $E$ whose fiber over each point $x$ is the tangent space at $x$ to the fiber copy of $M$ in which $x$ lies. We shall examine the degree to which the corresponding notion makes sense in the PL category. For instance, if the diagram above is now understood to represent a PL fibering, the PL $n$-plane bundle of tangents along the fiber is well defined. We see this by assuming without loss of generality that $B$, and therefore $E$, are themselves PL manifolds. Consider $D=\{(x, y) \in E \times E \mid \pi x=\pi y\}$. Let $\Delta: E \rightarrow D$ be the diagonal map embedding $E$ in $D$; then the normal (micro) bundle $\nu$ of $\Delta E$ in $D$ may be thought of as the "bundle of tangents to $E$ along the fiber." Note that on each fiber $M^{n}$ of $E, \nu \mid M^{n}$ is the tangent $n$-plane bundle of $M^{n}$. However, the question remains whether this PL $n$-plane bundle arises from a PL $(n-1)$-sphere (or PL $n$-disk) bundle. We shall see that, in general, this cannot be the case even when the tangent bundles to each fiber $M^{n}$ are quite naturally defined by PL $S^{n-1}$ bundles. Specifically, we have the following result.

THEOREM 1. There exist examples of $k$-vector bundles $V_{1}, V_{2}$ over $B$ such that (1) $V_{1}$ is equivalent to $V_{2}$ as a PL-disk bundle (so that the associated sphere bundles $S_{1}, S_{2}$ over $B$ are homeomorphic as fiber spaces over $B$ via $h: S_{1} \rightarrow S_{2}$ ).

(2) The $(k-1)$-vector bundles $T_{1}, T_{2}$ of tangents along the fibers of $S_{1}$ and $S_{2}$ do not admit a PL-disk bundle map $D\left(T_{1}\right) \rightarrow D\left(T_{2}\right)$ covering $h$.

Received by the editors August 22, 1986.

1980 Mathematics Subject Classification (1985 Revision). Primary 55R25, 55R60.

Key words and phrases. Vector bundle, PL-disk bundle, PL-sphere bundle, bundle of tangents along the fiber, bundle of $k$-frames.

Partially supported by NSF DMS 84-02595. 
This observation results from examining how the principal $O(n)$ bundle corresponding to an $n$-vector bundle $W \rightarrow C$ is built up in stages. If we choose $W_{1}$, $W_{2}$ to be PL equivalent and $W_{1}$, in fact, to be trivial as a vector bundle while $W_{2}$ is not, it is clear that the corresponding principal $O(n)$ bundles cannot even be fiber-homotopy equivalent. This elementary fact will imply the stated result.

1. The bundle of $n$-frames associated to an $n$-vector bundle. Consider an $n$-vector bundle $W$ over a paracompact Hausdorff space $X$, e.g. a countable simplicial complex. We may thus assume that the structure group is reducible to $O(n)$. We propose to study the associated bundles of $k$-frames, $k=1, \ldots, n$.

First, recall that to any such bundle $W$ there is associated a bundle $V_{k, n}(W)$ of $k$-frames $k=1,2, \ldots, n$ whose fiber at $x \in X$ is the space of orthonormal $k$-frames in the fiber $W_{x}$ of $W$ lying over $x$. The fiber of $V_{k, n}(W)$ is thus a copy of the standard Stieffel manifold $V_{k, n}=O(n) / O(n-k)$. It is well known that there is a tower of fiberings

(I)

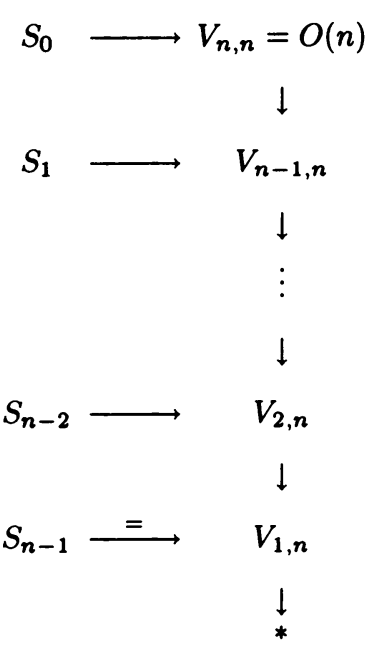

Correspondingly, there is, for $W$, a tower of fiberings

(II)

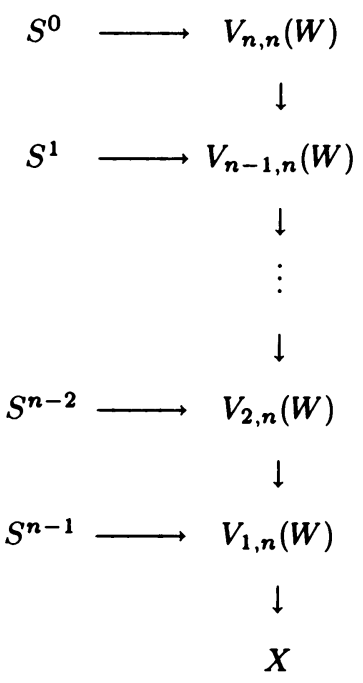


such that the composite map $V_{k, n}(W) \rightarrow X$ is just the bundle of $k$-frames associated to $W$.

We may describe these towers geometrically in a slightly nonstandard way as follows: To build up diagram (I), we can start with the $(n-1)$-sphere, obviously identifiable with $V_{1, n}$. If we then consider the tangent bundle $T\left(S^{n-1}\right)$ it is clear that $V_{2, n}$ may be identified with the subbundle of unit tangent vectors, i.e. $V_{2, n}$ is the $(n-2)$-sphere bundle associated to the tangent vector bundle of $S^{n-1}$. We continue in this manner inductively under the following hypothesis: suppose that we have obtained (for $k<n$ )

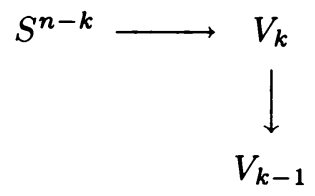

Let $T_{k}$ denote the $(n-k)$-vector bundle over $V_{k}$ of the tangents along the fiber $S^{n-k}$ 's. Then let $U_{k}$ be the unit $(n-k-1)$-sphere bundle associated to $T_{k}$. Then we claim that $U_{k}$ is identifiable with $V_{k+1, n}$ and that we thus have the standard fibering

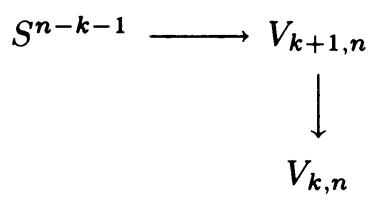

This claim may be verified by an easy exercise.

By a slight generalization, we may characterize diagram (II) similarly. That is $V_{1, n}(W)$ is quite obviously the $(n-1)$-sphere bundle over $X$ associated to $W$. It is then easily seen that $V_{2, n}$ may be described as follows: Let $T_{1}$ denote the bundle over $V_{1, n}(W)$ of tangents to the fiber $S^{(n-1)}$ 's of $V_{1, n}(W) \rightarrow X$. Let $U_{1}$ denote the $(n-2)$-sphere bundle associated to $T_{1}$. Then $U_{1}$ is canonically identifiable with $V_{2, n}(W)$.

Continuing inductively, we look at the fibering

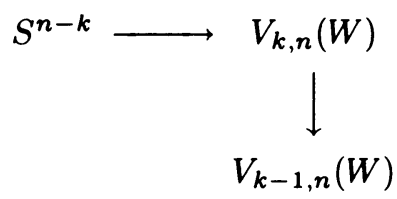

for $k<n$. Let $T_{k}$ now denote the $(n-k)$-vector bundle of tangents along the fiber for this fibering and $U_{k}$ the associated $(n-k-1)$-sphere bundle. Then $U_{k}$ is canonically identifiable with $V_{k+1, n}(W)$.

Again, the verification of this claim is an easy exercise.

2. Proof of Theorem 1. Consider a pair of $n$-vector bundles $W_{1}, W_{2}$ over $X$ such that $W_{1}$ is the trivial $n$-vector bundle and $W_{2}$ is not, yet $W_{1}$ is PL-equivalent to $W_{2}$. Such examples are known to exist. For instance, Milnor in [M, §9] gives examples of stable vector bundles over the 2-cell complex $X=S^{4 n-1} \cup_{q} e^{4 n}$ which are nontrivial, yet become trivial when regarded as topological microbundles. (Here $n>1$ and $q$ denote the attaching map of degree $q$, where $q$ is a prime dividing $\left(2^{2 n-1}-1\right) \cdot \operatorname{num}\left(B_{n} / n\right)$. $B_{n}$ denotes the $n$th Bernoulli number and $\operatorname{num}\left(B_{n} / n\right)$ is 
the numerator of the rational number $B_{n} / n$ when the latter is expressed in lowest terms.) Since, according to $[\mathbf{K}]$, microbundles are topological fiber bundles, and since $X$ is at least 6-connected, the result of Kirby and Siebenmann [KS] implies that the topological triviality of these vector bundles entails their PL triviality as well. Moreover, since they are stable bundles, we may consider that they are equivalent as PL-disk bundles (see $[\mathbf{H W}]$ ).

Assume the negation of Theorem 1, viz. assume that whenever we have $k$-vector bundles $V_{1}, V_{2}$ over $B$ which are PL-disk bundle equivalent (via $h: V_{1} \rightarrow V_{2}$, say), then the induced PL-equivalence of associated $(k-1)$-sphere bundles $h^{\prime}: S_{1} \rightarrow S_{2}$ will be covered by a PL-disk bundle map $g^{\prime}: D\left(T_{1}\right) \rightarrow D\left(T_{2}\right)$, where $T_{1}$ and $T_{2}$ are the $(k-1)$-vector bundles over $S_{1}, S_{2}$ respectively consisting of tangents along the fiber of

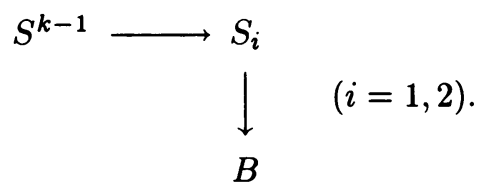

Now consider diagram (II) for $W=W_{1}$ and $W=W_{2}$ respectively. Given the hypothetical assumption above, let us assume that we have connected the two versions of diagram (II) with PL equivalences up to the $k$ th stage, i.e.

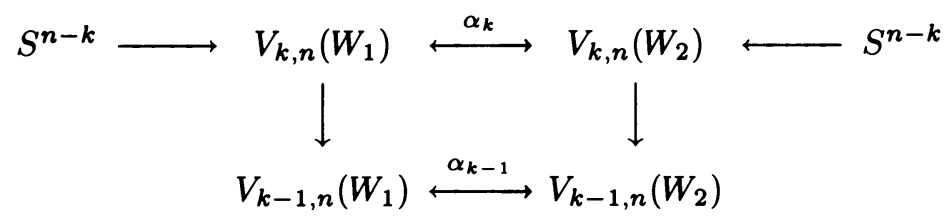

where the PL homeomorphism $\alpha_{k}$ is a PL-sphere bundle map covering $\alpha_{k-1}$. We may use $\alpha_{k-1}$ to regard $V_{k-1, n}\left(W_{2}\right)$ as a single space (call it $B$ ). Abbreviate $V_{k, n}\left(W_{i}\right)$ as $V_{i}, i=1,2$. Then, by our hypothesis (now letting $T_{i}$ denote the bundles of tangents along the fiber to $V_{i} \rightarrow B$ ), we have a PL-disk bundle map $\beta_{k+1}: D\left(T_{1}\right) \leftrightarrow D\left(T_{2}\right)$ covering $\alpha_{k}$. Letting $U_{i}, i=1,2$, be the associated $(n-k-$ 1)-sphere bundle and $\alpha_{k+1}$ the equivalence induced by $\beta_{k+1}$, we see that we have now extended the equivalence of the two versions of diagram (II) up to the $(k+1)$ st level, i.e., we now have

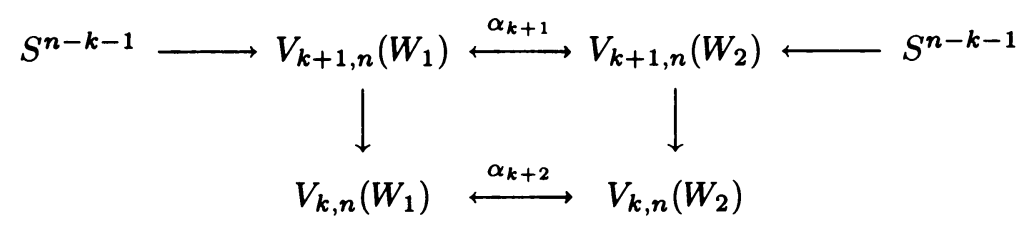

It ultimately follows, then, that we have a diagram (ignoring intermediate stages)

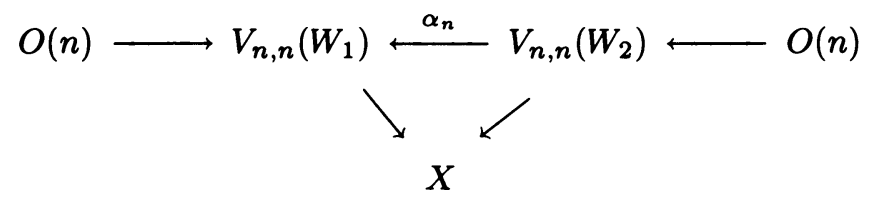

where $\alpha_{n}$ is a homeomorphism fiberwise and, in fact, a map of locally trivial fiber spaces. Recalling now, that $W_{1}$ is in fact trivial, $V_{n, n}\left(W_{1}\right) \rightarrow W_{1}$ surely admits a section. But therefore, so does $V_{n, n}\left(W_{2}\right) \rightarrow X$. Since this last is the principal 
$O(n)$ bundle associated to $W_{2}, W_{2}$ must be trivial as well, contradicting the known nontriviality of $W_{2}$.

It follows, then, that the hypothesis we introduced must have been false at some stage. I.e., for some $k$, there was a diagram

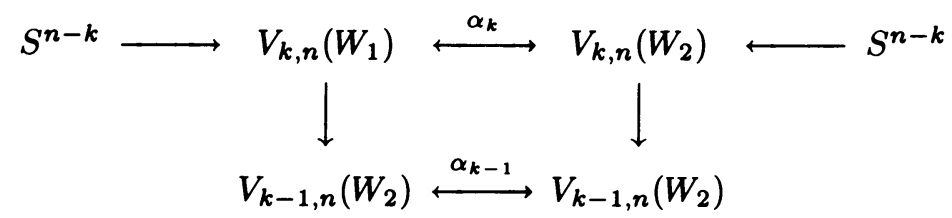

where the tangent bundles along the fiber of $V_{k, n}\left(W_{i}\right) \rightarrow V_{k-1, n}\left(W_{i}\right), i=1,2$, could not be connected by a PL-disk bundle map covering $\alpha_{k}$. This proves Theorem 1 .

REMARK. We note, as an easy corollary, that the proof of Theorem 1 is readily modified to handle other possible bundle theories to which vector bundles forgetfully map, viz, PL-block bundles, topological bundles, and spherical fiber spaces.

COROLLARY 2. In the statement of Theorem 1, let the assumed PL-equivalence of (1) be replaced by block-bundle equivalence, topological equivalence, or fiberhomotopy equivalence. (In the last case, the homeomorphism between $S_{1}$ and $S_{2}$ becomes a homotopy equivalence.) Let "PL-bundle map" in (2) be replaced correspondingly by block-bundle map, topological bundle map or map of spherical fiberspaces. Then Theorem 1, as altered, remains true.

\section{REFERENCES}

[HW] A. Haefliger and C. T. C. Wall, Piecewise linear bundles in the stable range, Topology 4 (1965), 209-214.

[K] J. Kister, Microbundles are fibre bundles, Ann. of Math. (2) 80 (1964), 190-199.

[KS] R. Kirby and L. Siebenmann, On the triangulation of manifolds and the Hauptvermutung, Bull. Amer. Math. Soc. 75 (1969), 742-749.

[M] J. Milnor, Microbundles. I, Topology 3 (Suppl. 1) (1964), 53-80.

DePaRtMent of MAThematics, RUTGers UNIVERSity, NeW BRUNSWICK, NeW JeRSEY 08903 